\title{
Crohn's disease in a child
}

Case report

Sathiadas $\mathrm{MG}^{1}$, Arunath $\mathrm{V}^{1}$, Ginige Anusha ${ }^{2}$

\section{Introduction}

Inflammatory bowel disease (IBD) in children is poorly understood and both Crohn's disease and ulcerative colitis are characterized by unpredictable exacerbations and remissions. The most common time of presentation is preadolescent/adolescent (1). IBD is classified according to the age of presentation as paediatric onset ( $<17 \mathrm{yrs}$ ), early onset ( $<10 y r s$ ), very early onset ( 6 yrs) and infant /toddler onset (0-2 yrs). (2)It has been regarded as a disease primarily occurring in Western populations. Although it was earlier considered rare in Asia, a number of recent reports suggest an increase in the incidence and the prevalence in this region as well.(3) We report a case of Crohn's disease in a six year old child from Jaffna.

\section{Case report}

A 6 years old boy was admitted with a history of 20 days of fever and one week of left sided abdominal painwhich worsened with meals. He also had 4-5 episodes of diarrhea mixed with blood and mucous on several occasions. He had lost $1.5 \mathrm{~kg}$ since the onset of the disease and experienced loss of appetite. He experienced a similar illness two months ago where he had watery loose stools.

There was no contact history of similar episodes, nocontact history of TB and family history of IBD. The family had good hygienic practices and did not consume food from eating places.
On examination, his weight for age was less than $3^{\text {rd }}$ centile, height for age was at $50^{\text {th }}$ centile and BMI for age was less than $5^{\text {th }}$ centile. He was febrile, pale and had generalized abdominal tenderness. The investigations showed a haemoglobin of $9.2 \mathrm{~g} / \mathrm{dL}$. Other red cell indices and blood picture revealed normocytic normochromic anemia, neutrophil leukocytosis and thrombocytosis The ESR was 120 and CRP was 100.9. Renal and liver profiles were normal except serum albumin which was $24 \mathrm{mg} /$ dLUrine, stool and CSF examinations were normal. Serum amylase and LDH levels were normal as well. TB PCR, bone marrow examinations were negative. USS abdomen revealed mesenteric lymphadenopathy which was less than $7 \mathrm{~mm}$ in size. CT abdomen showed small hypo echogenic areas in spleen.

The inflammatory markers and the fever did not respond to intravenous cefotaxime, Meropenam and Amikacin. Due to the persistent nature of the fever and blood stained stools an endoscopy was done which showed inflammatory changes and multiple biopsies were taken. Biopsy revealed goblet cell depression, crypt abscess formation and small granulomas. Appearances were those of a patchy active chronic colitis which was compatible with Crohn's disease. (Figure 1)

The Paediatric Crohn's Disease Activity Index (PCDAI) was used to assess the level of disease and to monitor

Figure 1: Microscopic appearances

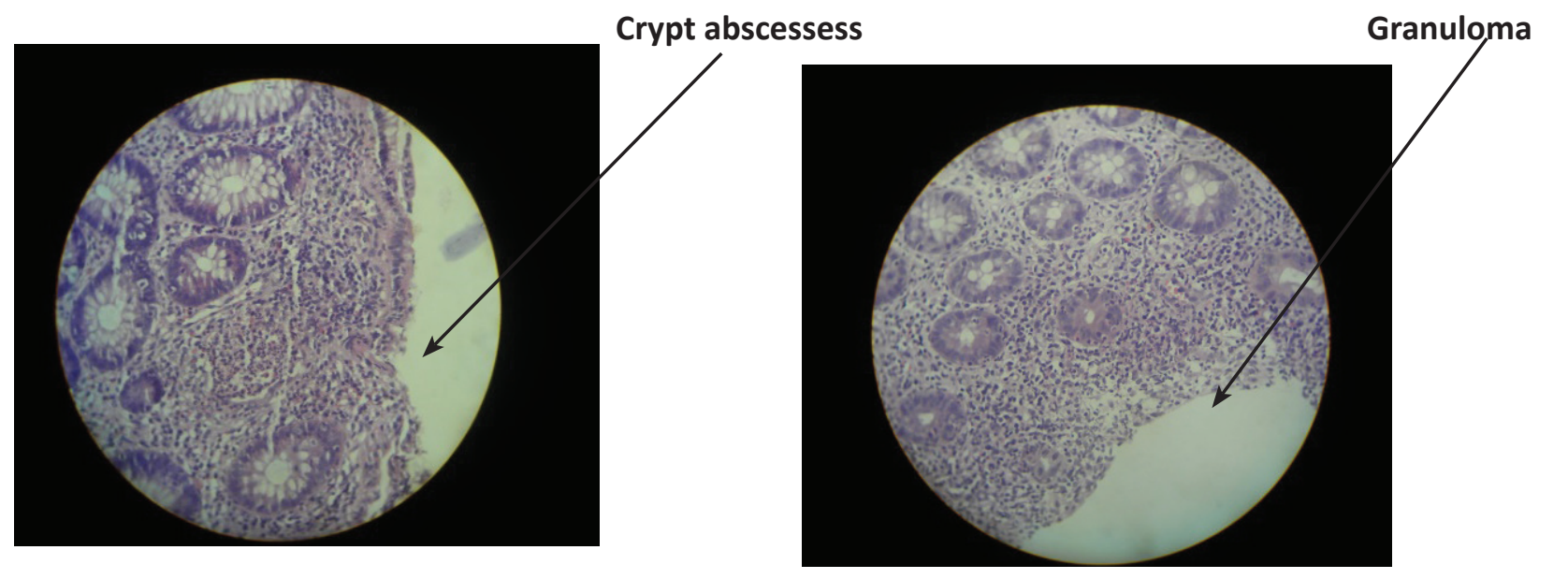

${ }^{1}$ Faculty of Medicine, Jaffna, ${ }^{2}$ Teaching Hospital, Jaffna 
the disease process. (4) The initial score was 42.5 which indicated severe disease at diagnosis and the score became 10 with treatment. A response to disease activity was seen with pulse methyl prednisolone followed by prednisolone with improvement of clinical features, reduction of inflammatory markers (ESR 70) and improvement in the serum albumin $(34 \mathrm{mg} / \mathrm{dl})$. A flare up of disease occurred 2 weeks after discontinuing steroids and Azathioprine was commenced. He remains in remission with a good clinical response and normal inflammatory markers. The plan is to continue azathaioprine and the disease activity will be monitored by suing the PCDAI.

\section{Discussion}

Inflammatory bowel disease (IBD) includes ulcerative colitis and Crohn's disease, which are chronic inflammatory diseases of the gastrointestinal tract. The underlying aetiology and pathogenesis of IBD remains largely unknown, but are thought to result from an interaction between genetic susceptibility, environmental factors such as gut microbial and the host immune response. Reasons for the increasing incidence of IBD in Asia are probably multi-factorial, and may at least partly relate to changing dietary habits. (4)

The clinical score is simple to perform, sensitive to changes in clinical status, reproducible by different observers, and specifically designed to evaluate inflammatory bowel disease in children and adolescents. The clinical score is a useful adjunct in the management of children with chronic inflammatory bowel disease and can be used in prospective studies of various therapeutic modalities.This multi-item instrument (PCDAI) consists of four general fields: history, physical examination, growth parameters, and common laboratory tests. The PCDAI differs from the adult Crohn Disease Activity Index (CDAI) primarily in adding growth parameters and laboratory measures and by decreasing the weighting of subjective parameters and eliminating antidiarrheal agents as a variable. Disease activity score of $<10$ indicates remission, with 10-27.5 - mild, 30-37.5 - moderate and >40 - severe

Treatment aims are to induce and maintain clinical remission, optimize nutrition, define bone status, optimise growth and pubertal progress and minimize drug adverse effects. The strategy of early introduction of immunomodulatory and biological therapies is to induce deep remission (long-term intestinal healing without relapse) particularly in high-risk patients (severe endoscopic or perianal disease, poor response to induction therapy, extensive, structuring or penetrating disease, marked growth retardation. Exclusive enteral nutrition (EEN) for 6-8 weeks, using a whole protein formula is the first-line therapy to induce remission in children with active CD.
Corticosteroids can be used to induce remission in $C D$, if EEN is not possible or the patient does not respond to therapy. Prednisolone is given orally $(1 \mathrm{mg} / \mathrm{kg}$, maximum: $40 \mathrm{mg} /$ day, tapering over 8-10 weeks) for moderate/ severe active luminal CD. Anti-tumour necrosis factor (TNF) therapy is also recognized as induction therapy for selected patients with moderate to severe disease. Maintenance of remission is achieved with Thiopurines, methotrexiate, Anti TNF- $\alpha$-Antibody and aminosalicilates. (5) Patients with late-onset paediatric, severe/extensive or structuring / penetrating CD have increased the risk for bowel surgery. Emergency surgical interventions are indicated in circumstances such as severe hemorrhage, intestinal perforation and toxic megacolon.

\section{Conclusion:}

Blood and mucous diarrhea should alert the treating paediatrician regarding IBD, diagnosing and monitoring of the disease is vital for long term prognosis. The management of paediatric CD has evolved significantly over recent years with evidence-based guidelines now in place to assist day-to-day practice.

\section{References:}

1. Kliegman, Stanton, St Geme, Schor, Nelson Textbook of Paediatrics; $20^{\text {th }}$ Edition, p1819-31, Elsevier publication, ISBN 978-81-312-4370-1

2. Levine A, Griffiths A, Markowitz J, Wilson DC, Turner $D$, et al, Pediatric modification of the Montreal classification for inflammatory bowel disease: The Paris classification; Inflammatory Bowel Diseases. 2011:17(6):1314-1321.doi: 10.1002/ibd.21493. Epub 2010 Nov 8.

3. Eric I. Benchimol MD, PhD, Kyle J. Fortinsky BSc,Peter Gozdyra MA,Meta Van den Heuvel MD, Johan Van Limbergen MD, PhD,Anne M. Griffiths MD; . Epidemiology of pediatric inflammatory bowel disease: A systematic review of international trends. Inflammatory bowel disease.2011:17(1):423-439, January 2011:doi: 10.1002/ ibd.21349

4. Hyams J, Markowitz J, Otely A, Rosh J, Mack D, et al; Evaluation of the Pediatric Crohn Disease Activity Index: A Prospective Multicenter Experience. Journal of Pediatric Gastroenterology and Nutrition. 2005:41:416-421. Lippincott Williams \& Wilkins, Philadelphia: Available from: http://journals.lww. com/jpgn/Fulltext/2005/10000/Evaluation_of_the_ Pediatric_Crohn_Disease_Activity.7.aspx

5. Kammermeier J, Morris M, Garrick V, et al. Management of Crohn's disease. Archives of Disease in Childhood 2016;101:475-480. doi:10.1136/ archdischild-2014-307217. 